\title{
Effective Leadership Style in Extractive Industry: A Case Study of Emerging Companies in Mozambique
}

\author{
Anivaldo Chevane ${ }^{1 *}$, Fadil Citaku ${ }^{1}$, Claudio Violato ${ }^{1}$, Yawar Hayat \\ Khan $^{1}$, Don Zillioux ${ }^{1}$, Lumturie Preteni Citaku ${ }^{1}$, Marianne \\ Waldrop $^{1}$ \\ ${ }^{1}$ Academy of Leadership Sciences, Switzerland \\ *Corresponding author: Anivaldo Chevane: achevane@gmail.com
}

\section{OPEN ACCESS}

Citation: Chevane A., Citaku F., Violato C., Khan Y.H., Citaku L.P., Waldrop M.(2017)Effective Leadership Style in Extractive Industry: A Case Study of Emerging Companies in Mozambique. Open Science Journal 2(4)

Received: $18^{\text {th }}$ September 2017

Accepted: $11^{\text {th }}$ October 2017

Published: $21^{\text {st }}$ November 2017

Copyright:@ 2016 This is an open access article under the terms of the Creative Commons

Attribution License, which permits unrestricted use, distribution, and reproduction in any medium, provided the original author and source are credited.

Funding: The author(s) received no specific funding for this work

Competing Interests: The author have declared that no competing interests exists.

\section{Abstract:}

This research aimed assessing the effective leadership recommended in Extractive Industries (ExI) in Mozambique considering its rapid growth. Primarily the hypothesis was that new entrants which in the case of ExI will preferably perform beyond expectations when subjected to directing and coaching leadership styles. Additionally, the rapid growth of the industry demands a lesser experienced internal workforce as domestic regulation limit the hiring of external workforce, while demanding high productivity. The situational approach is that leadership adapt his style according to the situation of the follower. Based on the results, it was not possible reject the null hypothesis; however, it was possible to prove that coaching style was positively correlated and statistically significant with motivation and motivation with performance. So indirectly, alternative hypothesis was accepted, thus, the leader's performance in the proposed environment significantly correlates with coaching style. The interaction plot also shows the tendency of positive performance with increased directing and participating. Delegating style, although performs as other styles in small quantities, when increased, leads to both demotivation and deficient performance. The experienced workforce was demotivated; potentially caused by the mismatch between leadership style and the context of the follower; with leaders lacking supportive style when it was needed. The new entrants' willingness was high, probably because most are derived from low paid environments while ExI are highly attractive. This willingness however depreciated with time due to poor supportive style. 
Keywords: Effective Leader, Extractive Industries in Mozambique, Low skilled workforce, New entrants, Productivity, Situational Leadership, Skill shortage.

\section{Introduction}

The nature of extractive industry (ExI) is notoriously complex. Despite its associated complex processes, it has the privilege of being integrated by multifaceted and diverse professionals in terms of culture, sector, and degree of academic skills, as well as, experiences. Mozambique is facing a rapid growth in ExI with numerous multinational companies starting production in diverse commodities. However, workers have limited skills or lack experience in this kind of industry, in fact, most are novices. Despite skill shortage of domestic worker (Salinger\&Caroline, 2014), Mozambique Mining and Labour Laws reinforces maximizing hiring national citizens and is highly restrictive for hiring external workers.

These international companies have made their investment and are striving for ramp-up production to recover their investment in a short period of time despite domestic workers' lack of skill. Most authors, e.g. (Northouse, 2010; Armstrong, 2009), argued that it is the leader's sole responsibility to inspire followers to do their best and to perform beyond expectations, while also promoting change within organizations.Situational leadership approach, teaches the effective behaviours to be adopted by leader in function of development level of the follower (i.e. competency and commitment).While competence is the knowledge and ability to perform a specific task, commitment is the level of willingness as well as confidence for performing that task.

\section{Problem statement, hypothesis, and relevance}

As stated during the introduction, extractive companies face a trade-off with the requirement of ramping their production to recovering their investments in a short time, maintaining efficiency, while attempting to adhere to the mandate of maximizing hiring internal workers in spite of their low skill in extractive industry. This research is important because in spite of these identified trade-offs, this subject represents a little researched topic in Mozambique especially for extractive industry. The research may be timely considering that the extractive industry is incipient in Mozambique and may serve as an exciting area for future research. The hypothesis according to existing literature,(e.g. Northouse, 2010; Bass, 1990; Vecchio, 1987) is that, in the proposed environment better performance will be dependent with directing and coaching leaders 'behaviour. 


\section{Leadership theories}

Leadership is a relationship process whereby leaders influence the followers to achieve common goals (Northouse, 2010). Leaders can adopt different leadership styles in their role depending on the followers' abilities, role complexity and structure, environmental situation, member relations and, most importantly, the leader's personal ability. In his book (Northouse, 2010), summarizes different perception of different leadership styles.

While the trait approach recognizes that some leaders have specific innate attributes or what leaders are, the skill approach is centred on what leaders can accomplish based on their position level.Top level leaders must have the ability to work with ideas (i.e. holding vision) while, lower level must be technically skilled (working with things).

Contingency and situational approach is contingent on matching the leader to the situation. The difference is that, while the contingency approach is static, the situational approach offers flexibility. According to (Fiedler, 1967) in (Armstrong, 2009 , p. 5), the contingency approach "is not meaningful to speak of an effective leader or an ineffective leader [...], we can only speak of a leader who tends to be effective in one situation and ineffective in another". As for the situational approach, we talk about the effective leader no matter the situation.

Transformational or Charismatic Leadership is defined by (Armstrong, 2009), a leadership perspective that explains how leaders change teams by creating, communicating, and modelling a vision for team work and organizations and inspiring the employees to strive for that vision. (Armstrong, 2009), added that this differs from transactional leadership that is somewhat confused with management which is only concerned with achieving current objectives by exchanging things of values. In this situation, the transformation occurs because is best interest of subordinate's agenda. Charisma, although necessary, is not sufficient for transformational leadership; however, it isa crucial element for promoting change and movement.

Leader - follower exchange approach focuses on how we handle relationships with followers, either formal or informal. Informal relations are restricted to contractual agreements in out-group relationship;formal relations are based on mutual trust, respect, liking, and reciprocal influence in partnership phase.

\section{Situational Leadership}

The situational leadership style is the behaviour pattern that is enacted by the person who intends to influence others according to their developmental level.

According to(Klatt \& Hiebert, 2001), there is not one right way to lead. For effective leadership, every circumstance demands a unique kind of leadership, where continuous development and influence for those being led matches their level of skill/competency and motivation/commitment. According to (Blanchard, 1985), in his modified model SLII, the situational styles can be classified into four categories comprising intermediate stages of directive and supportive behaviours. Similarly, there are four developmental levels that respond best to unique leadership styles. Subordinates at higher development levels exhibit interest, 
confidence and have mastered enough skill to perform a job, while those at lower development levels demonstrate high motivation but lack the skill to accomplish the task.

Directing style is appropriate for those subordinates that are highly interested, though are unfamiliar with the specific task (i.e. low competence/ high commitment follower). As the fol-lower gains experience, he may still require the leader's approval or may quit when the task requires more in-depth problem solve skills, negatively effecting his motivation (i.e. some competence/low commitment). In instances like these is when the Coaching style is recommended. Supportive style is applicable for those followers who may respond favourably to facilitating behaviours from their leaders in light of having moderate competence, and highly variable commitment. Delegating style is applicable for those employees that have mastered the skills to do a specific task and developed a positive attitude regarding the task (i.e. high competence/ high commitment).

Whatever the situation is, for the leader to be effective, ac-cording to (Batool, 2013; Goleman, 2000), requires the ability to handle his and other's emotions in such way that produces desirable results. In other words, citing (Van Rooy \& Viswesvaran, 2004, p. 72), effective leaders are those who offer a "[...] set of abilities (verbal and non-verbal) that enable a person to generate, recognize, express, understand, and evaluate their own and others' emotions to guide thinking and action that successfully cope with environmental demands and pressures". These abilities define leaders' emotional intelligence and are argued to be dependent on personal (self-awareness and motivation) and social (selfregulation, empathy, and social awareness) competencies e.g. (Batool, 2013; Goleman, 2000; Van Rooy \& Viswesvaran, 2004; Goleman, 1998; Goleman, Boyatzis \& McKee, 2002). When those competencies are integrated into cognition, (Harms \& Credé, 2010) they are essential for communicating visionary messages (Goleman et al., 2002).

\section{Methodology}

A sample-based design selected participants from among professionals within extractive industries in different commodities especially from oil/gas industry, coal mining, heavy sands, metal and gems, and graphite mining; the Mozambican extractive industries that are experiencing ramp-up production. The primary source of data was acquired through the online questionnaire-based survey. An online survey method was used due to the participants being geographically dispersed.For quantitative assessment, a closed-ended question format was found to be appropriate. Nearly 100 professionals were invited to participate in the survey. Only $52 \%(\mathrm{n}=52)$ participants from those invited accepted and, $69.2 \%$ $(\mathrm{n}=36)$ of those successfully completed the survey and had their participation validated. The $30.8 \%(\mathrm{n}=16)$ participants with uncompleted responses, answered the general questionnaire, however, not leadership assessment.

The research questionnaire used, was adapted from that proposed by (Hersey, Blanchard \& Johnson, 1996) for situational leadership assessment. The questionnaire is composed of 12 questions with different situations and four answer-options corresponding the 4 leadership styles, behaviours, and attitudes 
for each situation. These 12 questions, are accomplied by questions that inquire about the respondent's position, age, experience, role, motivation, performance, size of the company as well as the number of people in the group. The scoring table also proposed by (Hersey et al., 1996) was used. All analysis was performed using Minitab 17 incorporating diverse robust statistic tools.

\section{Results}

\section{General results}

Among those who successfully completed the questionnaire (36), the majority, $47 \%$, were staff with bachelor degrees or above, followed by team leaders also with bachelor degrees or above $(25 \%)$, and senior leaders $(22 \%)$. Staff with $12^{\text {th }}$ grade or below were the smallest group with just $6 \%$.

Leaders' position, both senior and team leaders compose $47 \%$ while staff is marginally higher at $53 \%$. Most of the participants were young adults ranging from 19 to 35 years old $(70 \%)$, while adults 36 years and above were $30 \%$. $81 \%$ of participants were involved in the organization in its initial stages (between exploration and ramp-up mining or extraction) of the projects, while, $19 \%$ were in stable production industries. More than half (53\%) reported to have between 5 10 years of experience, $25 \%$ had less than 5 years, and $22 \%$ had above 10 years.

The majority were in coal mining industries $(67 \%)$, followed by oil and gas extraction industries with $14 \%$. According to Mozambican classification by national institute of statistic (INE), mining industries are mostly medium to large organizations (MLE), with over 50 employees. Those compose $78 \%$ of the sample, while $22 \%$ fall into small to medium organizations (SME). $47 \%$ of participants reported being in teams of less than 5 co-workers, 33\% from 10 to 20 and $19 \%$ were members of large teams with more than 20 workers.

Based on (Hersey et al., 1996) situational assessment, $46 \%$ of the participants indicated their propensity for applying coaching style in diverse situations. Directing and participating were statistically similar, with participating style high with $28 \%$ of $20 \%$ for directing. The similarity of means between directingand participating was later confirmed by analysis of variance (ANOVA) Tukey Method with the same mean variance group. Delegating style was not a popular amongst the participants, with only $6 \%$.

Intuitively, there are similarities found between the motivation and performance curves. While $63.9 \%$ reported continuing to grow in motivation, $61.1 \%$ reported that their group performance is good. $8.3 \%$ of the group members declared declining motivation, while equivalent poor performance also measured at $8.3 \%$. Reports of high motivation were $11.1 \%$ and similarly $11.1 \%$ reported that their performance excels. Sufficient performance with $19.4 \%$ is probably related with $16.7 \%$ of those who declared that there are demotivated, Fig. 1. 


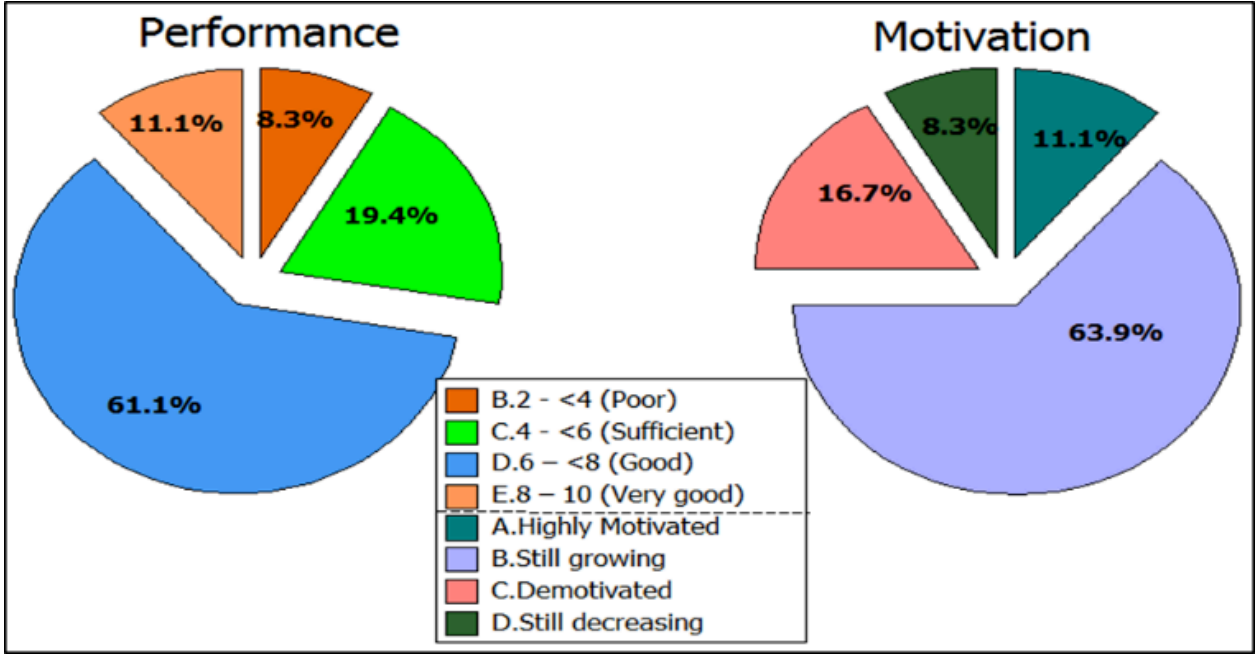

Fig. 1: Pie chart of performance and motivation assessment, showing similarities between the proportion of performance grouping as function of the motivation group.

\section{Interaction plots}

Different scenarios of interaction plots were run between the situational factors. For quantitative evaluation, each option code was attributed to its highest rank numerical values as seen in Table 1. 
Table 1: Matrix code for the factors

\begin{tabular}{|c|c|c|c|}
\hline Parameter & Option & Option Code & Numeric value \\
\hline \multirow{4}{*}{ Level } & Senior leader & A & 1 \\
\hline & Team Leader & B & 2 \\
\hline & Staff (Bachelor or above) & C & 3 \\
\hline & Staff (High School-grade 12 or below) & D & 4 \\
\hline \multirow{4}{*}{ tompany Dimensio } & 0-20 employees & A & 20 \\
\hline & 21-100 employees & B & 100 \\
\hline & 101-500 employees & C & 500 \\
\hline & $>500$ employees & $\mathrm{D}$ & 1000 \\
\hline \multirow{4}{*}{ Age } & 18 to 25 years & A & 25 \\
\hline & 26 to 35 years & B & 35 \\
\hline & 36 to 45 years & C & 45 \\
\hline & $>45$ years & D & 50 \\
\hline \multirow{2}{*}{ Role } & Administrative (e.g. HR, financial, suppliers, IT...) & A & NA \\
\hline & Operation (e.g. geologists, mining engineers, operators, process engineers, surve & $B$ & NA \\
\hline \multirow{4}{*}{ Team size } & $>5$ subordinates/coworkers & A & 5 \\
\hline & 5 to 10 subordinates/coworkers & B & 10 \\
\hline & 11 to 20 subordinates/coworkers & C & 20 \\
\hline & $>20$ subordinates/coworkers & $D$ & 30 \\
\hline \multirow{5}{*}{ Comodity } & Coal & A & NA \\
\hline & Oil/Gas & $B$ & NA \\
\hline & Graphite & C & NA \\
\hline & Metals and gems & $D$ & NA \\
\hline & Heavy sands & $\mathrm{E}$ & NA \\
\hline \multirow{4}{*}{ Project Phase } & Exploration & A & 1 \\
\hline & Pre-mining/extraction & B & 2 \\
\hline & Rump up mining/extraction & C & 3 \\
\hline & Stable mining/extraction & $D$ & 4 \\
\hline \multirow{4}{*}{ Experience } & 0 to $<2$ years & A & 2 \\
\hline & 2 to $<5$ years & B & 5 \\
\hline & 5 to $<10$ years & C & 10 \\
\hline & $>10$ years & $D$ & 15 \\
\hline \multirow{5}{*}{ Performance } & $0-<2$ (Very poor) & A & 2 \\
\hline & $2-<4$ (Poor) & $B$ & 4 \\
\hline & 4 - <6 (Sufficient) & C & 6 \\
\hline & $6-<8($ Good $)$ & D & 8 \\
\hline & $8-10$ (Very good) & E & 10 \\
\hline \multirow{4}{*}{ Motivation } & Highly Motivated & A & 15 \\
\hline & Still growing & B & 10 \\
\hline & Still decreasing & C & 5 \\
\hline & Demotivated & D & 0 \\
\hline
\end{tabular}


Based on the interaction plots, Directing and Coaching style was preferable among the lower level environments (i.e. staff with grade 12 or below). Participating was at mid-level while delegating was at the highest level. Initially, it was assumed that level would be directly related to experience (e.g. less experience and lower level more directive, while, highly experienced and upper levels more delegating); however, this was only minimally true on lower level as less experienced employees also preferred directing style. Coaching style was dominant in highly experienced environments, while, participating and delegating were at medium to less experienced environments.

The level and experience mismatch on the expected leadership curve is probably due to leaders at the upper level who are less experienced leaders, although they reported more than a year of experience, and preferred to be directive rather than delegating, as would be expected.

In fact, it has been industrial practice that highly skilled technicians are easily promoted to leadership positions without required preparation. People who are promoted to the leadership position because of their adept technical skill without required leadership preparation or innate leader traits tend to resist being delegative regardless of their role or follower circumstances. These leaders normally lack confidence in other's ability to accomplish the task alone and prefer to be directive even with those who demonstrate mastery in skill and commitment. The lack of confidence in others is potentially associated with lack of emotional intelligence, despite their high level of cognitive ability. The performance of those who are expected to delegate but prefer to be directive is questionable and may lead to a "lose-lose" proposition, i.e. losing a proficient technician to gain an even worse leader. The leader's age was technically similar with level.

As expected, directing style was preferred in larger companies or larger groups, while, supportive style was preferred in the smaller companies or smaller group settings. Is not clear however, why coaching style was preferred in small team set-tings and ironically, why delegating style was preferred in larger organizational settings. Generally, small companies are easier to lead and are less bureaucratic than larger organizations. Additionally, the development of the follower is in general quicker, making the delegating style preferable and therefore, more appropriate.

Departments taked with operational functions prefer participating and delegating styles, while administrative departments prefer directing style. This is probably because administrative tasks are less complex, repetitive, and highly regulated when compared to operational tasks that demand proficiency in highly specialized skills.

Initial phases of projects, for instance exploration and pre-striping phases, demand more directing styles; while stable mining demands a more delegating style. This is expected in initial stages of ExI projects, where more are new workers are without experience. In general, coaching style followed by participating style (i.e. both sum $74 \%$ ) were most preferable. Increased coaching together with participating style lead to motivation of the group member. Increased delegating style most often leads to demotivation, although in small doses had similar performance to the other styles, while stable for directing style.

Improved performance is observed for leaders with $12^{\text {th }}$ grade education or below and senior leaders; while performance remains stable for leaders at 
intermediate levels, small companies or small teams, youth employees and those who are less experienced at early stages of projects. The correlation between performance and motivation is clear; high motivated teams realize excellent performance.

The better performance of less experienced workforce when observed on the interaction plot Fig. 2, is high due to their level of willingness, not competence. Their level of willingness is probably increased by both their large learning curve as well as attractive incentives. Most organizations work force is derived from low paid/low productivity environments and workers find ExI attractive and opportune to boost their career experience. However, this perception is only true in the beginning of a career. Once the follower gains experience and his basic needs increase over time, his motivation is no longer sustained by these two factors and requires additional development and incentives, such as social group relations, rewards, etc.

The skilled or more experienced followers are mostly a demotivated group and according to (Blanchard, 1985) the supportive approach is most appropriate for this situation as they are operating within the third development level and are likely to exhibit resistance.

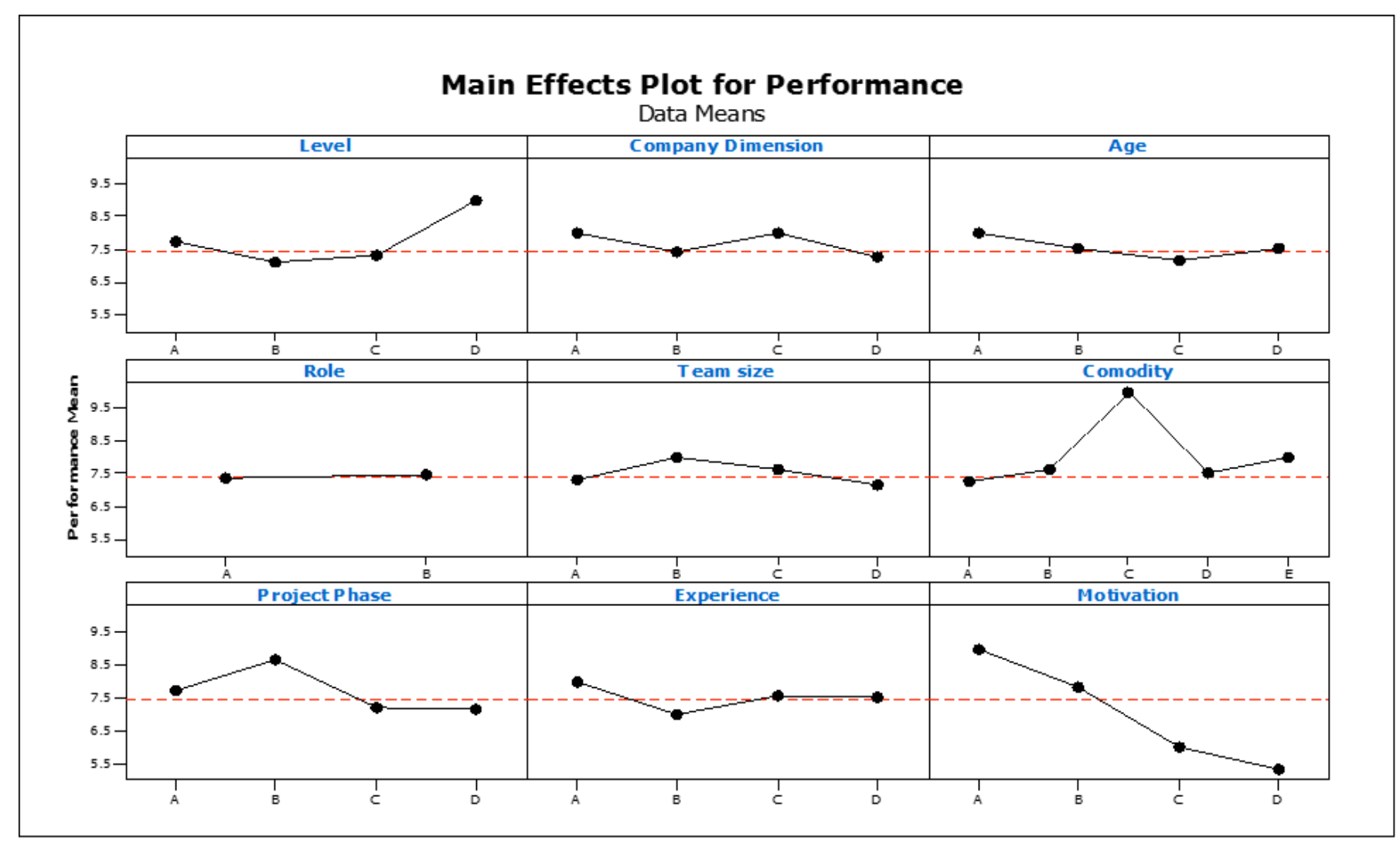

Fig. 1: Main effect plots of performance with distinct factors, highlighting that better performance is observed on those aging young and highly motivated workers with lesser experience.

General trend shows the tendency of increasing performance with increasing directing and participating style, while coaching was highly variable, although, at the high-performance side. Increasing delegating style led to poor performance. 
As the trend of performance is similar to that observed in motivation when correlated with leadership styles, it can be concluded that poor performance was due to the follower's low commitment which potentially caused a mismatch between leadership style and follower development level and behaviour.

Through observing the performance of delegating leadership style, it was noted that the performance of team members, although rejecting delegating style is well above average (i.e. similar to other styles) when regulated. Although followers, prefer coaching to moderate supportive and directive styles, they also require some freedom to perform tasks that they experience when subjected to delegating style, Fig. 3.

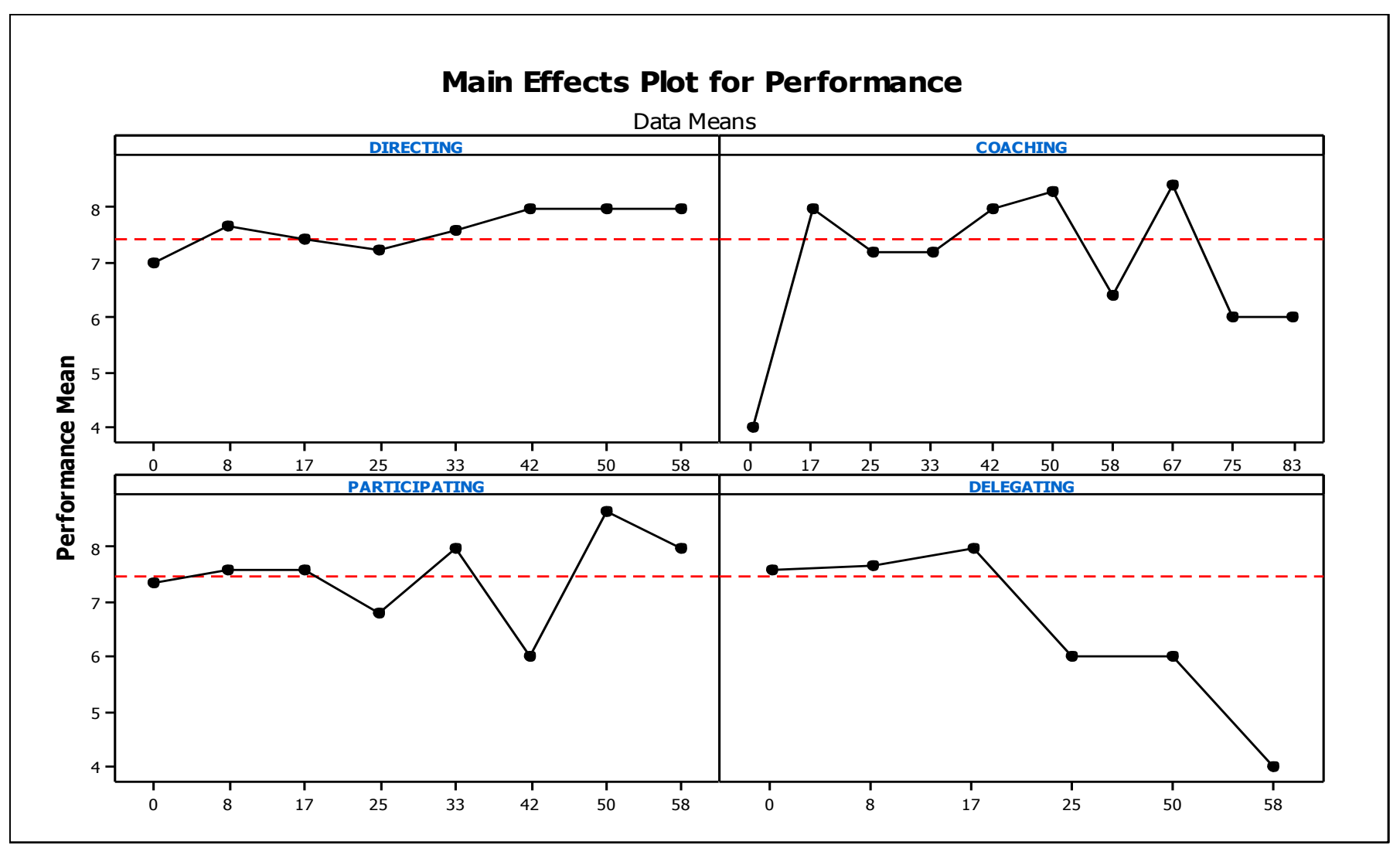

Fig. 2: Main effect plots of performance in function of different leadership styles, showing the tendency of poor performance with increased delegating style, as well as, increased performance with increased directing and participating style, while highly variable performance in coaching style.

\section{Pearson correlation and p-value hypothesis tests}

While main effect plots offered a visual interaction of the factors, Pearson correlation and p-value indicate whether there is correlation between factors and what is statistically significant of the correlation. Pearson correlation equals zero meaning that there is no correlation between factors, while, $\mathrm{p}$-value $\geq 0.05$ means that if the correlation is available it's not statistically significant between variables. 
To test the hypothesis proposed in this study, Pearson correlation (first condition) and p-value (second condition) will both be used so that:

- $\mathrm{H} 0$ - Null Hypothesis, i.e. $\mathrm{R}=0 \& \mathrm{P}$-value $\geq 0.05$ : The leader's performance in the proposed environment will not be strongly correlated with dimension of directing and coaching leadership style.

- $\mathrm{H} 1$ - Alternative Hypothesis, i.e. $\mathrm{R} \neq 0 \& \mathrm{P}$-value $<0.05$ : The leader's performance in the proposed environment will be strongly correlated with dimension of directing and coaching leadership style.

Where $\mathrm{R}$ represents the Pearson correlation factor.

Table 2: Matrix plot of Pearson correlation and P-value showing that first condition (R) tests is verified within all variables, however, only few passed second condition (P-value). Highlight is the negative and statistically significant correlation for delegating style with performance, as well as, significance of coaching style with motivation and motivation with performance

\begin{tabular}{|c|c|c|c|c|c|c|c|c|c|c|c|c|}
\hline Designation & & Level & $\begin{array}{c}\text { Company } \\
\text { Dimension }\end{array}$ & Age & $\begin{array}{c}\text { Team } \\
\text { number }\end{array}$ & $\begin{array}{l}\text { Project } \\
\text { phase }\end{array}$ & $\begin{array}{c}\text { Years of } \\
\text { experience }\end{array}$ & Performance & Motivation & Directing & Coaching & Participating \\
\hline Pearson correlation(R) & \multirow{2}{*}{ Company Dimension } & 0.151 & & & & & & & & & & \\
\hline P-Value & & 0.379 & & & & & & & & & & \\
\hline Pearsan correlation( $(R)$ & \multirow{2}{*}{ Age } & -0.591 & 0.064 & & & & & & & & & \\
\hline P-Value & & 0.000 & 0.710 & & & & & & & & & \\
\hline Pearsan correlation(R) & \multirow{2}{*}{ Team number } & -0.379 & 0.067 & 0.202 & & & & & & & & \\
\hline P-Value & & 0.023 & 0.699 & 0.238 & & & & & & & & \\
\hline Pearson correlation( $(R)$ & \multirow{2}{*}{ Project phase } & -0.234 & 0.593 & 0.450 & 0.069 & & & & & & & \\
\hline P-Value & & 0.170 & 0.000 & 0.006 & 0.688 & & & & & & & \\
\hline Pearsan correlation(R) & \multirow{2}{*}{ Years of experience } & -0.377 & 0.219 & 0.589 & 0.209 & 0.470 & & & & & & \\
\hline P-Value & & 0.023 & 0.200 & 0.000 & 0.220 & 0.004 & & & & & & \\
\hline Pearsan correlation $(R)$ & \multirow{2}{*}{ Performance } & 0.025 & -0.101 & -0.070 & -0.040 & -0.188 & 0.079 & & & & & \\
\hline P-Value & & 0.885 & 0.560 & 0.684 & 0.816 & 0.273 & 0.649 & & & & & \\
\hline Pearsan correlation $(R)$ & \multirow{2}{*}{ Motivation } & -0.255 & -0.135 & 0.164 & -0.025 & -0.096 & 0.188 & 0.665 & & & & \\
\hline P-Value & & 0.133 & 0.432 & 0.338 & 0.883 & 0.577 & 0.271 & 0.000 & & & & \\
\hline Pearsan correlation $(R)$ & \multirow{2}{*}{ Directing } & -0.168 & 0.057 & -0.119 & 0.213 & 0.123 & -0.100 & 0.127 & 0.028 & & & \\
\hline P-Value & & 0.327 & 0.742 & 0.491 & 0.212 & 0.474 & 0.561 & 0.460 & 0.870 & & & \\
\hline Pearsan correlation $(R)$ & \multirow{2}{*}{ Coaching } & -0.140 & -0.029 & 0.130 & -0.184 & 0.064 & 0.342 & 0.136 & 0.331 & -0.368 & & \\
\hline P-Value & & 0.416 & 0.867 & 0.449 & 0.282 & 0.713 & 0.041 & 0.429 & 0.049 & 0.027 & & \\
\hline Pearson correlation $(R)$ & \multirow{2}{*}{ Participating } & 0.243 & -0.140 & 0.091 & -0.194 & -0.297 & -0.170 & 0.038 & 0.085 & -0.535 & -0.299 & \\
\hline P-Value & & 0.154 & 0.415 & 0.596 & 0.257 & 0.078 & 0.322 & 0.824 & 0.623 & 0.001 & 0.076 & \\
\hline Pearson correlation(R) & \multirow{2}{*}{ Delegating } & 0.077 & 0.148 & -0.156 & 0.249 & 0.142 & -0.141 & -0.371 & -0.580 & 0.043 & -0.569 & -0.231 \\
\hline P-Value & & 0.656 & 0.390 & 0.364 & 0.143 & 0.408 & 0.412 & 0.026 & 0.000 & 0.802 & 0.000 & 0.175 \\
\hline
\end{tabular}

The table of testing shows that the correlation between all variables passed the first condition (i.e. Pearson correlation); revealing that there is some level of correlation (negative or positive) between the factors; however, the statistical significance of the correlation (i.e. second condition) is only verified between some variables.

There are three statistically significant correlations with level (age, team size and experience); all of them with negative correlation. However, the level was inverted on the ranking table, with low numeric values corresponding to high level and vice-versa. Therefore, negative correlation should be interpreted as positive and vice-versa. This means that young adults were better suited for leading large groups with relatively more experience. The company size was 
statistically significant as expected from the main effect interaction plot with project stage (i.e. from exploration to stable production). The correlation is positively indicating that a highly-experienced workforce is associated with larger organizations.

As expected, age was significantly and positively correlated with project phase and experience. Early stages of organizational development typically have a young, less experienced workforce, while more mature organizations have highly experienced and an older workforce.

While large companies were operating at more developed levels, the small companies were in early development stages. This is expected as exploration stages only hold small teams of geologists, while, at mining stages multifunctional tea msare necessary.

Motivation and performance ranked higher on correlation and showed to be statistically significant, which is consistent with the body of literature discussing leaders' performance. It was not possible to prove the significance of directive and coaching style relative to performance. However, unquestionably, exercising the delegating style led to both demotivation and poor performance.

Although the null hypothesis could not be rejected directly for coaching style, it could be rejected (i.e. there is statistically significance between coaching and performance) indirectly considering that coaching style was statistically significant with motivation. Motivation was found to be the principal ingredient for performance.

\section{Effective Leadership (proposed model)}

As expected and discussed in the literature review, there is no standard formula to be effective. The leader must adapt his leadership style according to the given situation. Generally, based on general findings, it is concluded that the most appropriate style for the discussed situation is coaching style. The coaching style is highest ranked, and the only style that can statistically account for performance.

The directing and participating styles were statistically similar in rank (with participating best ranked), but significance tests placed directing style second. So, the recommended order based on statistical significance tests is high coaching and moderate directive to less supportive and discouraged delegative style.

Different situations demand different combinations of styles, so while the coaching style most contributed to leadership style, the specific combination of factors leads to performance beyond expectations. Fig. 4, shows that the combination of young age which is highly motivated can surpass by far the more mature worker with extensive experience.

The combination of level and motivation is second of the top 8 most affecting performance. The correlation of performance and level is positive (negative considering the inversion of level rank), this means that better performance is observed on those youth, at initial levels when highly motivated. Coaching style at mid-level and directive style at younger ages at mid-level are also within top 8 performance. 

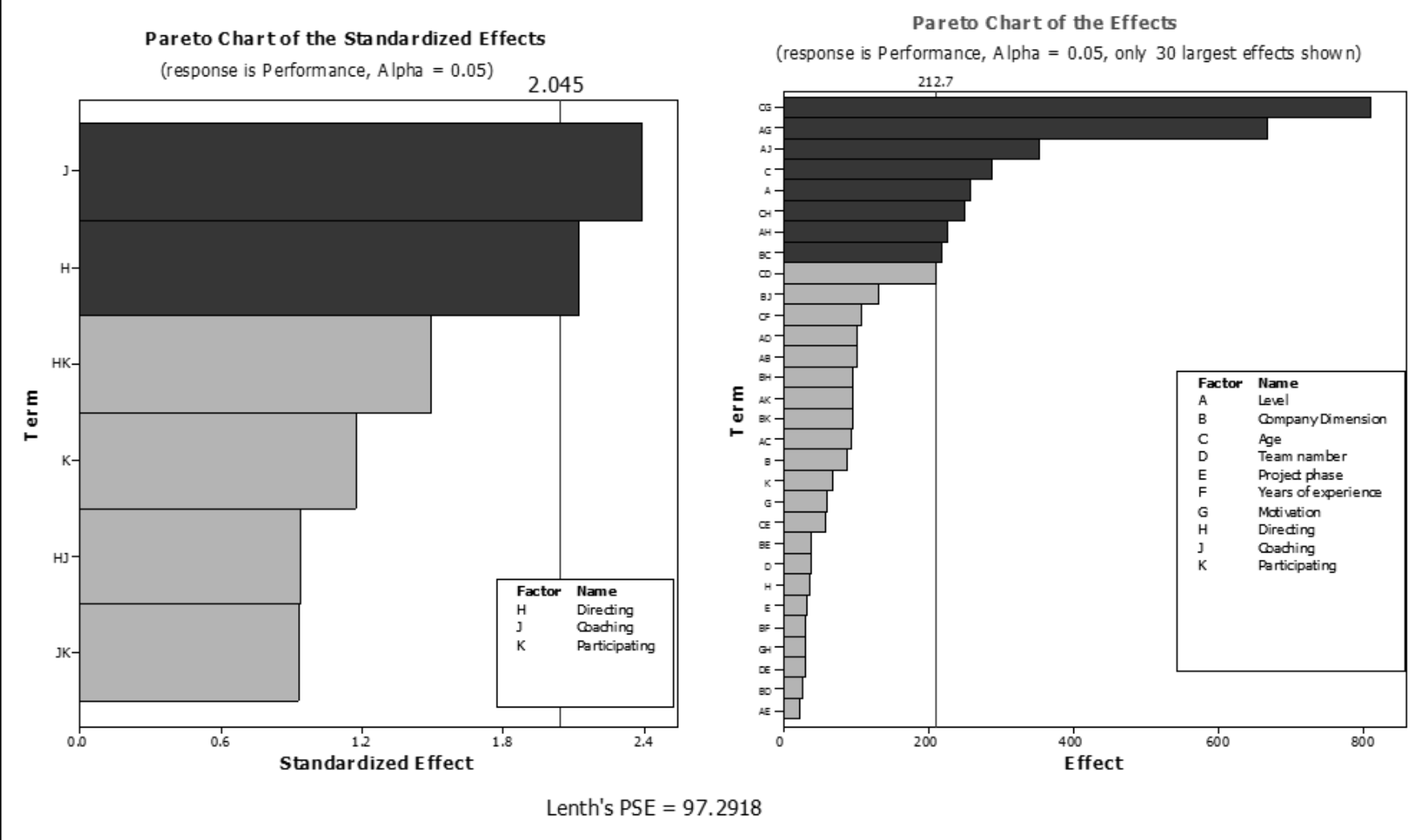

Fig. 3: Interaction main effect pareto for performance. Coaching and directing leadership styles are most affecting performance. Combination of age, level with motivation ranking top 8 best performance groups.

\section{Limitations}

The limitation of this study is associated with the sample size and confidence level of the survey. The targeted sample size was 100, in order to secure a margin of error between 5 to $10 \%$ at $95 \%$ of confidence level. However, due to low participation rate $(52 \%)$ as well as the rate of completion $(69.2 \%)$, the valid sample size obtained was 36 . At the observed sample size, the margin of error is approximately $16 \%$ with $95 \%$ of confidence level.

Another limitation of the study was related to the assessment of performance and motivation from the respondents. The performance and motivation rank was relative to each respondent, making interaction analysis ambiguous as is not based on the same assumptions of defining the different level of motivation and performance. However, performance and motivation was highly correlated, minimizing the effect of the probable ambiguity.

A statistically meaningful relationship seen in the factors does not imply that a cause-effect relationship exists. It merely tests the statistical variation of given factors and its influence on the variation of the other factors. 


\section{Conclusions and recommendations}

Despite limitations raised and considering consistency of the results, the following conclusions and recommendations can be drawn:

In general coaching style followed by participating style (i.e. both sum 74\%) were most preferable. The directing and participating styles were statistically similar in rank with participating ranking highest in performance.

Increased coaching style and moderate directing and participating led to motivation of group members, while increased delegating led to demotivation. When regulated, however, delegating style led to the same levels of performance observed on the other best ranked styles. This is likely because although followers prefer coaching to moderate supporting and directing styles, they also require a minimum level of freedom offered by delegating style on their tasks as part of exercising their confidence level.

Operational workers preferred participating and delegating styles, while administrative workers preferred directingstyle. This is probably because administrative tasks are less complex and more repetitive when compared to operation tasks that demand specific and exceptional skills.

As expected, initial phases of projects, like during exploration and pre-striping where there are more inexperienced workers, demand more directive styles while stable mining demands a more delegating style. Small companies are easy to manage and less bureaucratic. Additionally, the development of the follower is generally faster where the delegative style is more appropriate. While large companies at more mature stages, the small companies were at initial stages. This is expected as exploration stages only holds small teams, while, at mining stages a multifaceted team is required.

Considering that most workers in Mozambique are from low paid/low productivity levels, the shift to ExI which is more attractive will lead to higher motivation in first years which in turn contributes to better performance. But as observed, high motivation decreases with time and supportive leadership is most appropriate to sustain their level of motivation. The mismatch of leadership style to the followers' development level leads to demotivation/low performance of high experienced workers.

As expected, age was significantly and positively correlated with project phase and experience. Early stages of the organization have younger, less experienced workforce, while mature stages hold, highly experienced with relatively mature workers.

Motivation and performance ranked higher on correlation and were statistically significant, which is consistent with existing literature regarding leaders' performance. It was not possible to prove the significance of directing, coaching or participating styles relative to performance. However, it was indisputable that exercising delegating style led to both demotivation and poor performance.

Although the null hypothesis could not be rejected directly for coaching style, it could be rejected (i.e. there is statistically significance between coaching and performance) indirectly considering that coaching style was statistically significant with motivation. Motivation was found to be the principal ingredient for performance. 
Young workers which are typically highly motivated can surpass by far the mature worker with renowned experience. The combination of age and motivation is the second of 8 most affecting performance. The correlation of performance and level is positive (negative considering the inversion of code rank), this means that better performance is observed on those youth, at initial levels highly motivated. Coaching style at mid-level and directing style at young age of mid-level are also within top 8 performance.

Companies must have their plan for leadership succession to properly prepare their future leaders.

Leadership can be learned, identifying, and defining the central competencies of leadership, however, remain elusive. There is a lot of literature written about leadership, but the robust research in this regard is lacking. One exception makes the study of (Çitaku, Violato, Beran, et al., 2012). These researchers have developed a leadership competencies model, empirically investigating the core leadership competencies. This model comprises 63 leadership competencies within five domains (1. Social responsibility; 2. Innovation; 3. Self-management; 4. Task Management; 5. Justice orientation). This empirical model of leadership competencies can be employed to develop curricula of training programs to teach and develop leadership competencies in any domain, including Extractive Industry.

Nonexistence or poor succession leadership planning leads to poorly selected leaders, only based on their expert technical skills. Although technical abilities are essential, leaders must have a high level of emotional intelligence. More practically, it is like a football team. The team leader must be technical and tactical skilled. While technical is knowledge both cognitive (intelligence) and crystalized ability (experience); tactical is sense of awareness of self as well as with who you are dealing with.

Despite consistency of the results, future similar research is recommended, with both performance and motivation assessed by researcher criteria to prove that respondents' motivation and performance are robust. In general, people have more affinity to reveal low motivation than to reveal poor performance.

\section{References:}

Armstrong, R. (2009). Armstrong's Handbook of Management and Leadership: A guide to managing for results (2nd ed.). London: British Library Cataloguing.

Bass, B. M. (1990). From Transactional to Iransformational Leadership: Learning to Share the Vision. $3,18,19$.

Batool, B. F. (2013). Emotional Intelligence and effective leadership (Vol. 4). Superior University.

Blanchard, K. H. (1985). SLII: A situational approach to managing people. Escondido, CA: Blanchard Training Development.

Çitaku, F., Violato, C., Beran, T., \& etal. (2012). Leadership competencies for medical education and healthcare professions: population-based study. BMJ Open. doi:10.1136/ bmjopen-2012-000812

Fiedler, F. E. (1967). A Theory of Leadership Effectiveness. NY: McGraw-Hill.

Goleman, D. ( 1998). Working with Emotional Intelligence. New York, NY: Bantam Books.

Goleman, D. (2000). Leadership that gets results. 78-90. Harvard Business Review.

Goleman, D., Boyatzis, R., \& McKee, A. (2002). Primal leader-ship: Realizing the power of emotional intelligence.

Harms, P. D., \& Credé, M. (2010). Emotional Intelligence and Transformational and Transactional Leadership: A Meta-Analysis. Journal of Leadership \& Organizational Studies, 17(1) 5 -17. doi:DOI: $10.1177 / 1548051809350894$ 
Hersey, P., Blanchard, K. H., \& Johnson, D. (1996). Management of Organizational behaviour: Utilizing human resources. (7th ed.). (P. Hall, Ed.) NJ: Upper Saddle River.

Klatt, B., \& Hiebert, M. (2001). The Encyclopaedia of Leadership: A Practical Guide to Popular Leadership Theories and Techniques. NY: McGraw-Hill Companies. doi:ISBN 0-07-136308-4.

Northouse, P. G. (2010). Leadership: Theory and practice (5th ed.). Thousand Oaks CA: Sage.

Salinger, L., \& Caroline, E. (2014). Mozambican Labor Markets In The Face Of A Natural Resource Boom - What Potential Impacts of Dutch Disease? Maputo: USAID/SPEED. doi:EDH-I-00-0600004-00/13

Van Rooy, D., \& Viswesvaran, C. (2004). Emotional intelligence: A meta-analytic investigation of predictive validity and nomological net. Journal of Vocational Behaviour, 65 (2004) 71-95. doi:10.1016/S0001-8791(03)00076-9

Vecchio, P. ( 1987). Situational leadership theory: An examination of prescriptive theory. Journal of applied Psychology, 72, 3, 444 